\title{
Variability in grading diabetic retinopathy from stereo fundus photographs: Comparison of physician and lay readers
}

\author{
ROY C. MILTON, ${ }^{1}$ JAMES P. GANLEY, ${ }^{2}$ AND RODNEY H. LYNK ${ }^{1}$ \\ From the National Eye Institute, National Institutes of Health, Bethesda, Maryland; ${ }^{1}$ and the \\ Division of Ophthalmology, University of Arizona Medical Center, Tucson, Arizona ;2 USA
}

SUMmary Two physicians and two lay readers were trained according to a detailed protocol in the grading of 17 lesions found in diabetic retinopathy by evaluation of stereo fundus photographs according to a modified Airlie House classification. Intraobserver and interobserver variability of these readers was assessed by two methods: weighted kappa, and frequency of agreement within one grade.

In general, physician readers were found to be less variable on replicate readings than were lay readers, and had slightly better agreement with each other than with the lay readers. The physiological significance of the direction and magnitude of the difference between physician and lay reader variability for individual lesions was often uncertain. Assessment of contribution to disagreement by individual readers was possible and permits future training directed at reducing disagreement to acceptable values.

Stereo fundus photography has become widely accepted as a means of documenting ocular pathology in therapeutic trials such as the collaborative Diabetic Retinopathy Study (Kupfer, 1973; Diabetic Retinopathy Study Research Group, 1976), in epidemiological studies such as the Framingham Eye Study (Kahn, 1973), and in clinical follow-up of patients as for suspected malignant melanoma lesions (Ganley and Comstock, 1973). The use of photographs offers several advantages over clinical evaluation, although these two methods must be considered complementary rather than competitive. First, photographs provide greater opportunity to standardise evaluation techniques and to assess and control variability. Secondly, photographs are permanent records of pathology which permit systematic re-evaluation by other observers and by other grading criteria.

Individual physician-patient relationships are still best served, logistically and professionally, by each physician making his own clinical assessment of pathology according to accepted standards and techniques. Concern for standardisation of evaluation and for control of variability increases in research

Address for reprints: Dr Roy C. Milton, Office of Biometry and Epidemiology, National Eye Institute, National Institutes of Health, Bethesda, Maryland 20014, USA which emphasises group or population characteristics. This research, using statistical or epidemiological methods together with clinical evaluation, is appropriate when studying diseases considered to have multiple causality, with each cause being less than completely deterministic.

Ophthalmological studies of this kind, with several thousand patients perhaps followed up through repeated examination, frequently have large numbers of photographs to be evaluated. The logistics of grading these photographs raises several important considerations. Are sufficient ophthalmologists available to grade these photographs according to protocol requirements of standardised training and evaluation? Are ophthalmologist readers an efficient use of a highly skilled manpower resource? Should each photograph be graded more than once? By different graders? What is meant by accuracy, when no independent, objective, standard evaluation exists? What measures of variability are useful and adequately informative? How much grading variability is acceptable in general, or in a particular study? If non-physicians grade photographs, does this economic advantage reduce the quality of the readings? Success of studies involving photograph evaluation depends on answers to some or all of these questions. Although these questions 
are deceptively simple, it is not surprising that they may not have simple answers. The complexity of analysis reflects to some extent the difficulty in summarising complex study material.

In any study where observations are made, the potential for examiner variability is present and deserves considerable attention (Koran, 1975a, b). This includes variability in repeated observations by the same examiner, as well as variability of observations among different examiners (Kahn et al., 1975). To alleviate the economic and manpower problems when large numbers of photographs are to be evaluated, non-physician readers-often paramedical technicians-are at present employed to observe, grade, and record subjective and objective data. If lay readers without paramedical training can adequately evaluate and grade fundus photographs, this would further improve the economic and manpower considerations.

With the increasing use of non-physician readers, the potential for reader variability and reduced diagnostic accuracy is accentuated. Little information is available on the intraobserver (within observer) or interobserver (between observers) variability associated with reading fundus photographs by physician or non-physician readers, although the Diabetic Retinopathy Study is a potential source for such information.

The purpose of this paper is to present the results of a study designed, in a limited way, to compare lay readers with physicians for variability in grading stereo fundus photographs of patients with diabetic retinopathy. It is in a sense a pilot study, not intended to answer definitively all questions of interest with equal vigour; such an ambitious study may not be feasible. Rather, this study may provide insight into the magnitude and complexity of the problem, with examples of analytical methods and results which may be useful to those who develop and conduct large-scale studies using stereo fundus photographs.

\section{Materials and methods}

The classification and grading system for diabetic retinopathy presented at the Airlie House symposium (Davis et al., 1969), and further developed for the collaborative Diabetic Retinopathy Study was modified slightly for use in the present study. Standard stereo fundus photographs were used to classify and grade 17 different types of lesions found in diabetic retinopathy: haemorrhages and/or microaneurysms, hard exudates, soft exudates, venous abnormalities, arteriolar abnormalities, intraretinal microvascular abnormalities, arteriovenous nicking, macular oedema, neovascularisation near the disc, neovascularisation elsewhere, fibrous proliferation near the disc, fibrous proliferation elsewhere, plane of proliferation, retinal elevation, preretinal haemorrhage, vitreous haemorrhage, and intraretinal haemorrhage. Intraretinal haemorrhage was added to this study because of its frequent occurrence in diabetic retinopathy, and because lay readers were observed in the training session to misclassify intraretinal haemorrhage as either dot haemorrhages or preretinal haemorrhages.

Each reader was instructed to compare 'unknown' stereo photographs with a set of standard photographs of varying severity for each of the above 17 lesions. Depending on the severity of each lesion, each photograph was given a numerical grade. If a particular lesion was not present, the photograph was given a grade of zero for that lesion. If there was a question whether the lesion was present or absent a grade of 1 was recorded. Grades 2 through 5 were used to indicate increasing severity in relation to their respective standard photographs. A grade of 8 was used when the appropriate field for viewing a particular lesion was not present on the photograph being graded. A grade of 9 was used when it was not determinable whether a lesion was present or not; this might occur when large vitreous haemorrhages or massive vitreous proliferation obscured underlying retinal features.

A comprehensive protocol was developed for use in training the lay readers as well as the physician readers. Each lesion was described in detail and in language the lay readers could understand, and complete instructions were provided on how to grade the photographs according to the modified Airlie House classification.

A set of diabetic stereo fundus photographs was obtained from the Boston University Ophthalmology Department files for training purposes. For 3 weeks one of the investigators (R.L.) instructed the lay readers for approximately two hours each day, and they did further independent study daily for an additional two hours. Each lay reader had use of a Donaldson stereo viewer, teaching photographs, standard photographs, and a copy of the protocol and classification scheme. The training period was not extensive as the lay readers could spend only half of each day on training, while the remaining time was spent performing regular office duties. The physician readers went through a less extensive period of training, also becoming familiar with the classification scheme and the various lesions of diabetic retinopathy.

A set of diabetic stereo fundus photographs was obtained from the University of Wisconsin Department of Ophthalmology for use as 'unknowns'. These 97 photographs represented one or more of 
the seven standard fundus fields (Davis et al., 1969) from 14 patients with moderate to severe diabetic retinopathy. In addition 51 standard stereo photographs from 8 individuals without diabetic retinopathy were included as 'unknowns'. All photographs were taken with a fundus camera equipped with the Allen Stereo Separator.

Duplicate copies were made and randomly combined to form the full study group of 296 'unknowns'. Each stereo pair would then be evaluated independently of other fields in the same eye and of its duplicate pair. Each reader received the complete set of photographs in the same order but began at a different starting point. Since there were 17 conditions to be examined per stereo photograph, each reader made $5032(17 \times 296)$ readings.

Four readers participated in this study. Reader 1 was an opthalmologist with some experience in grading fundus photographs. Reader 2 was a physician with little ophthalmic background. Reader 3 was a secretary and Reader 4 a statistical clerk. Readers 1 and 2 knew the set of slides contained duplicates, but Readers 3 and 4 were not so informed, although at some point in the study they may have become aware of this fact.

The readers recorded their evaluations on coding forms which listed the various lesions to be evaluated, and which provided space for the reader's name, time reading began, time finished, and the identification numbers of the photographs being evaluated. When each reader finished a set of 10 photographs, the grades and photographs were returned and the next succeeding set obtained, thus preventing comparison with previous gradings. The data were keypunched and entered into the computer for editing and analysis. Minor inconsistencies in data collection discovered through editing were corrected.

\section{MEASURES OF VARIABILITY}

A variety of indices are available for assessing observer variability on categorical data described by ordinal scales (Landis and Koch, 1975). Choice of an index, while based partially on evaluation of suitability and informativeness for the problem at hand, is somewhat arbitrary since various properties of an index appeal differently to different users. Two methods were chosen for evaluation of intraobserver and interobserver variability in this study: weighted kappa (Cohen, 1968), based on the Rogot-Goldberg index of adjusted agreement (Rogot and Goldberg, 1966) (see appendix), and frequency of agreement within one grade.

In any study involving somewhat subjective observations some degree of interobserver and intraobserver variability is expected. Agreement within one grade was chosen to represent acceptable variability, while the complement-disagreement by more than one grade-was felt to represent a major degree of variability. For simplicity the few disagreements which involved non-ordered grades, i.e., 'wrong field' and 'not determinable', were omitted. The weighted kappa form of the Rogot-Goldberg index provides a measure which moderates the influence of artificially high agreement often observed when classifying rare phenomena, and which also readily permits statistical tests of significance. Weighted kappa $\left(\kappa_{\mathrm{w}}\right)$ may be described as the ratio of observed weighted agreement not accounted for by chance to possible weighted agreement not accounted for by chance. Thus $\kappa_{\mathrm{w}}=1$ indicates perfect agreement, $\kappa_{\mathrm{w}}=0$ chance agreement, and $\kappa_{w}<0$ less than chance agreement.

\section{Results}

The average time spent in grading all 17 lesions on each slide was 2.5 minutes per slide for the 2 physician readers, 5 minutes for Reader 3, and 11 minutes for Reader 4.

\section{INTRAOBSERVER VARIABILITY}

An example of intraobserver (within observer) variation in grading microaneurysms and/or haemorrhages is given in Table 1. Reader 1, on the first reading, graded 61 of the fields as having 'moderate' haemorrhages and/or microaneurysms as compared to the standard for that lesion; on the second reading 48 were graded as 'moderate'. Only 44 were graded 'moderate' on both readings. In this example all readers had difficulty in characterising the lesion as 'minimal' or 'moderate'.

Weighted kappa index of agreement. Assessment of intraobserver variability by weighted kappa was based on two-way frequency tables, such as Table 1. Values of weighted kappa measuring percent agreement $\left(100 \kappa_{\mathrm{w}}\right)$ between first and second readings for each reader for each of 16 lesions are given in Table 2 ( $\kappa_{\mathrm{w}}$ was not computed for vitreous haemorrhage owing to its rare occurrence).

Both physicians showed better agreement between first and second readings for 9 of the 16 lesions than did either lay reader. In seven instances the minimum physician agreement exceeded the maximum lay reader agreement by at least $10 \%$, with maximum exceedance of $34 \%$ occurring for retinal elevation, but these exceedances could have occurred by chance $(P>0 \cdot 20)$. For intraretinal haemorrhage both lay readers showed replicate agreement as good as or better than either physician.

For 15 of 16 lesions (all but intraretinal haemorrhage) the average physician agreement by weighted 
Table 1 Frequency of agreement between first and second reading of 148 fundus photographs for presence and severity of haemorrhages and/or microaneurysms, for 4 readers*

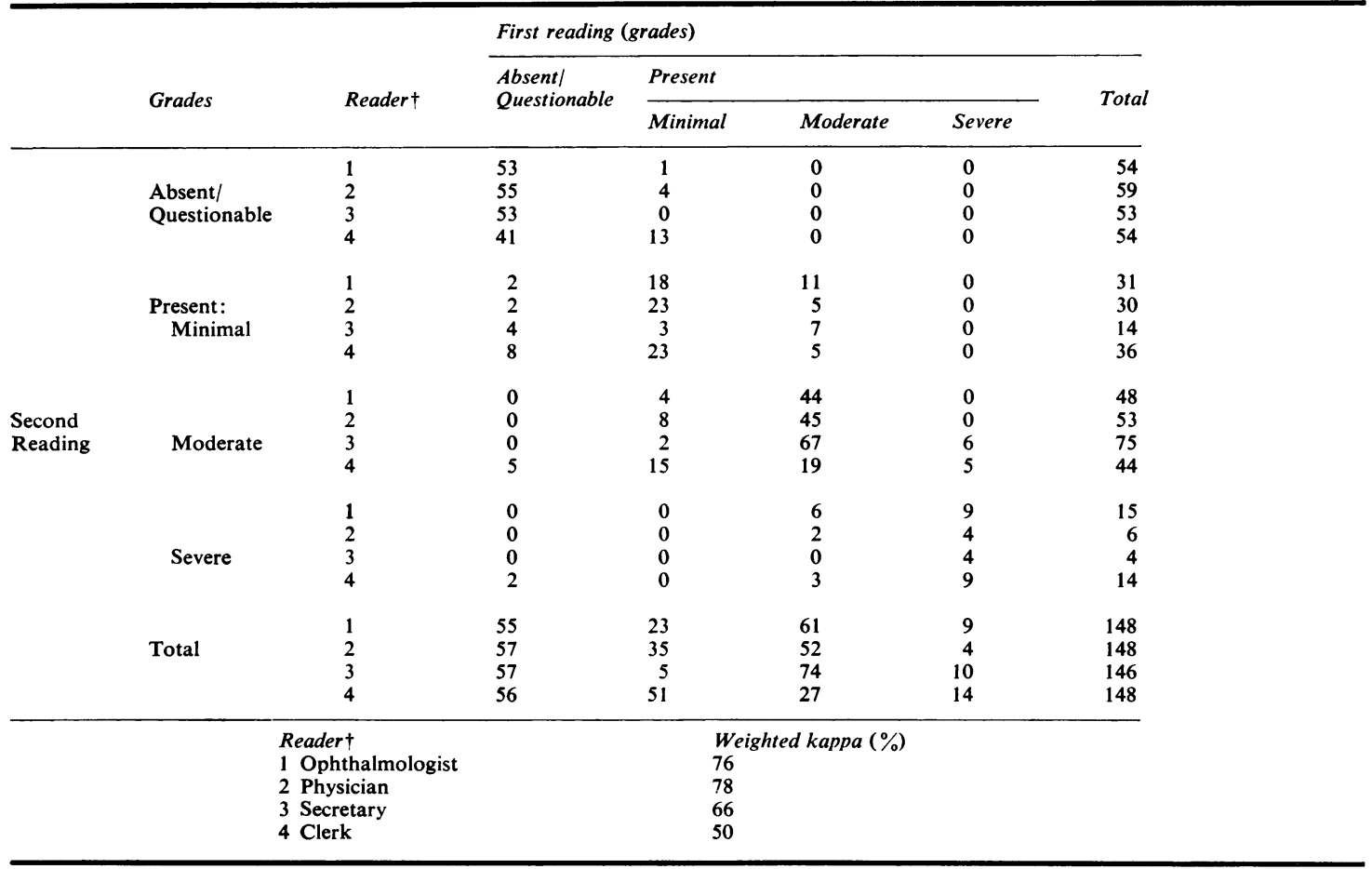

"Readings 'wrong field' and 'not determinable' are omitted.

kappa was greater than that for lay readers. In nine lesions the difference in weighted averages was at least $20 \%$, a statistically significant difference $(\mathrm{P}<0.02)$ for haemorrhages and/or microaneurysms and plane of proliferation. The maximum difference in averages was $49 \%$ for retinal elevation. Retinal elevation also had the greatest range of weighted kappa $(65 \%)$, and nine lesions had ranges greater than $25 \%$.

Rank comparison by Friedman's two-way analysis of variance* strongly suggests the readers differ in variability $\left(\chi_{3}^{2}=18.0, P<0.001\right)$. The magnitudes of the average ranks suggest that the physicians exhibited less variability in agreement than did the lay readers and further that the lay readers exhibited about equal variability.

Agreement within one grade. The frequency with which a reader agreed with himself within one grade on replicate readings is given in Table 2. For 9 of 17 lesions both physicians showed better agreement between first and second readings than did either

*For each of the 16 lesions, the indices for each abnormality were assigned ranks from smallest to largest ( 1 to 4$)$ for the readers. The null hypothesis is that the average rank over the 16 lesions (or, equivalently, the distribution of ranks for a reader) does not depend on the reader. lay reader. In two of these lesions (soft exudates and retinal elevation) the maximum lay reader agreement was at least $10 \%$ less than the minimum physician agreement. For intraretinal haemorrhage both lay readers showed equal or better agreement than either physician.

Analysis of variance by ranks of agreement percentages gives results similar to that for the index of agreement. Maximum range for percent agreement is $29 \%$ (fibrous proliferation of disc), and four lesions have ranges greater than $20 \%$. For 14 of 17 lesions the average lay reader percent agreement is less than that for physicians; in 5 cases the difference in averages is at least $10 \%$, with maximum of $24 \%$ for retinal elevation. Physician agreement varied from 86 to $100 \%$, with average agreement of $96 \%$ for each physician. Lay reader agreement varied from 71 to $100 \%$, with averages of 91 and $85 \%$ for Readers 3 and 4 , respectively.

\section{INTEROBSER VER VARIABILITY}

Weighted kappa index of agreement. Assessment of interobserver (between observers) variability by average weighted kappa is given in Table 3. Agreement was better between physicians than between 
Table 2 Intraobserver variability assessed by comparison of first and second readings of 148 fundus photographs for 17 diabetic retinopathy lesions among 4 graders by 'weighted kappa' and 'agreement within one grade'

\begin{tabular}{|c|c|c|c|c|c|c|c|c|}
\hline \multirow{3}{*}{ Lesion } & \multicolumn{4}{|c|}{$\begin{array}{l}\text { Agreement by weighted kappa }(\%) \\
\text { Readers }\end{array}$} & \multicolumn{4}{|c|}{$\begin{array}{l}\text { Agreement within one grade }(\%) \\
\text { Readers }\end{array}$} \\
\hline & \multicolumn{2}{|c|}{ Physicians } & \multicolumn{2}{|c|}{ Lay readers } & \multicolumn{2}{|c|}{ Physicians } & \multicolumn{2}{|c|}{ Lay readers } \\
\hline & 1 & 2 & 3 & 4 & 1 & 2 & 3 & 4 \\
\hline $\begin{array}{l}\text { Haemorrhages and microaneurysms } \dagger \\
\text { Hard exudates } \\
\text { Soft exudates } \dagger \ddagger \\
\text { Venous abnormalities } \\
\text { Arteriolar abnormalities } \\
\text { Intraretinal microvascular abnormalities } \\
\text { Arteriovenous nicking } \\
\text { Macular oedema* } \\
\text { Neovascularisation near the disc* } \\
\text { Neovascularisation elsewhere } † \\
\text { Fibrous proliferation near the disc* } \dagger \\
\text { Fibrous proliferation elsewhere } \dagger \\
\text { Plane of proliferation } \\
\text { Retinal elevation } \dagger \ddagger \\
\text { Preretinal haemorrhage } \\
\text { Vitreous haemorrhage } \\
\text { Intraretinal haemorrhage }\end{array}$ & $\begin{array}{r}76 \\
70 \\
82 \\
72 \\
74 \\
50 \\
66 \\
49 \\
65 \\
90 \\
100 \\
85 \\
59 \\
75 \\
100 \\
\text { nc } \\
88\end{array}$ & \begin{tabular}{r|}
78 \\
82 \\
85 \\
69 \\
64 \\
72 \\
76 \\
74 \\
83 \\
87 \\
100 \\
84 \\
76 \\
100 \\
81 \\
nc \\
94
\end{tabular} & $\begin{array}{l}66 \\
67 \\
54 \\
55 \\
64 \\
59 \\
66 \\
55 \\
60 \\
66 \\
69 \\
64 \\
76 \\
35 \\
66 \\
\text { nc } \\
93\end{array}$ & $\begin{array}{l}50 \\
61 \\
48 \\
76 \\
72 \\
36 \\
73 \\
49 \\
37 \\
56 \\
58 \\
62 \\
33 \\
41 \\
59 \\
\text { nc } \\
94\end{array}$ & $\begin{array}{r}98 \\
94 \\
100 \\
95 \\
95 \\
93 \\
95 \\
86 \\
95 \\
97 \\
100 \\
98 \\
93 \\
99 \\
100 \\
100 \\
94\end{array}$ & $\begin{array}{r}96 \\
97 \\
99 \\
89 \\
88 \\
97 \\
91 \\
90 \\
100 \\
95 \\
100 \\
96 \\
94 \\
100 \\
100 \\
100 \\
97\end{array}$ & $\begin{array}{r}97 \\
87 \\
84 \\
87 \\
95 \\
87 \\
94 \\
86 \\
100 \\
94 \\
95 \\
95 \\
92 \\
72 \\
97 \\
86 \\
97\end{array}$ & $\begin{array}{l}82 \\
96 \\
80 \\
89 \\
88 \\
75 \\
92 \\
76 \\
76 \\
82 \\
71 \\
91 \\
84 \\
78 \\
89 \\
98 \\
97\end{array}$ \\
\hline $\begin{array}{l}\text { Average } \\
\text { Average rank }\end{array}$ & $\begin{array}{l}75 \\
2 \cdot 8\end{array}$ & $\begin{array}{l}82 \\
3 \cdot 4\end{array}$ & $\begin{array}{l}63 \\
2 \cdot 0\end{array}$ & $\begin{array}{l}57 \\
1 \cdot 7\end{array}$ & $\begin{array}{l}96 \\
3 \cdot 2\end{array}$ & $\begin{array}{l}96 \\
3 \cdot 1\end{array}$ & $\begin{array}{l}91 \\
2 \cdot 1\end{array}$ & $\begin{array}{l}85 \\
1 \cdot 5\end{array}$ \\
\hline
\end{tabular}

*Based on only those (21) slides which include the appropriate field (disc or macula).

† Minimum physician agreement exceeds maximum lay reader agreement by at least $10 \%$, for weighted kappa.

$\ddagger$ Minimum lay reader disagreement exceeds maximum physician disagreement by at least $10 \%$, for disagreement by more than one grade. nc-Not computed, since lesion was extremely rare.

Table 3 Interobserver variability among 4 graders of 148 fundus photographs for 16 diabetic retinopathy lesions as measured by the average weighted kappa T

\begin{tabular}{|c|c|c|c|c|c|c|}
\hline \multirow[b]{2}{*}{ Lesion } & \multicolumn{6}{|c|}{ Average agreement between readers $(\%)$} \\
\hline & $\begin{array}{l}\text { Ophthall } \\
\text { Physician }\end{array}$ & $\begin{array}{l}\text { Secretary/ } \\
\text { Clerk }\end{array}$ & $\begin{array}{l}\text { Ophthall } \\
\text { Secretary }\end{array}$ & $\begin{array}{l}\text { Ophthall } \\
\text { Clerk }\end{array}$ & $\begin{array}{l}\text { Physician/ } \\
\text { Secretary }\end{array}$ & $\begin{array}{l}\text { Physician/ } \\
\text { Clerk }\end{array}$ \\
\hline Haemorrhages and microaneurysms $\ddagger$ & $66 *$ & $47 \dagger$ & 63 & 54 & 63 & 52 \\
\hline Hard exudates $\ddagger$ & $66 *$ & $39 \dagger$ & 54 & 62 & 47 & 59 \\
\hline Soft exudates $\ddagger$ & $67 *$ & 42 & 36 & $11 \dagger$ & 39 & 29 \\
\hline Venous abnormalities & 57 & $48 \dagger$ & 51 & $58 *$ & 52 & 54 \\
\hline Arteriolar abnormalities $\ddagger$ & 49 & 36 & $34 \dagger$ & 34 & 36 & $50 *$ \\
\hline Intraretinal microvascular abnormalities $\ddagger$ & 46* & 17 & 29 & 14 & $5 \dagger$ & 10 \\
\hline Arteriovenous nicking & 54* & 48 & 50 & $42 \dagger$ & 48 & 53 \\
\hline Macular oedema $\S$ & 22 & 19 & 17 & 46* & 30 & $1+$ \\
\hline Neovascularisation near the disc $\S$ & 21 & 41 & $69 *$ & 39 & 43 & $18+$ \\
\hline Neovascularisation elsewhere $\ddagger$ & 73* & 54 & 63 & $54 \dagger$ & 60 & 60 \\
\hline Fibrous proliferation near the disc $\S$ & 50 & 40 & $85 *$ & 39 & 56 & $13+$ \\
\hline Fibrous proliferation elsewhere & $66 *$ & $56 \dagger$ & 59 & 48 & 57 & 60 \\
\hline Plane of proliferation + & 43* & 18 & 14 & $9+$ & 41 & 18 \\
\hline Retinal elevation $\ddagger$ & $88 *$ & 19 & 36 & $-4+$ & 37 & 11 \\
\hline Preretinal haemorrhage & $80 *$ & 53 & 69 & 57 & 65 & $47+$ \\
\hline Intraretinal haemorrhage & 89 & 88 & 87 & $81+$ & 93* & 88 \\
\hline$* /+:$ & $10 / 0$ & $0 / 4$ & $2 / 1$ & $2 / 6$ & $1 / 1$ & $1 / 4$ \\
\hline
\end{tabular}

For each lesion, *indicates greatest agreement; tindicates least agreement.

$\ddagger$ Physician agreement exceeded lay reader agreement by at least $10 \%$

$\$$ Based on only those (21) slides which include the appropriate field (disc or macula).

TWhen comparing two readers who have two replicate readings, it is possible to compute two independent estimates of weighted kappa. The

average of these two values is a single measure of agreement which includes information from replication.

lay readers (column $1 v$. column 4) for all lesions except neovascularisation of disc, with maximum difference in agreement for retinal elevation (69\%). For 9 of 16 lesions physician agreement exceeded lay reader agreement by more than $10 \%$, a statisti- cally significant exceedance $(\mathrm{P}<0.05)$ for haemorrhages and/or microaneurysms, hard exudates, and plane of proliferation.

Agreement between physicians (column 1) exceeds the best agreement between either physician and a 


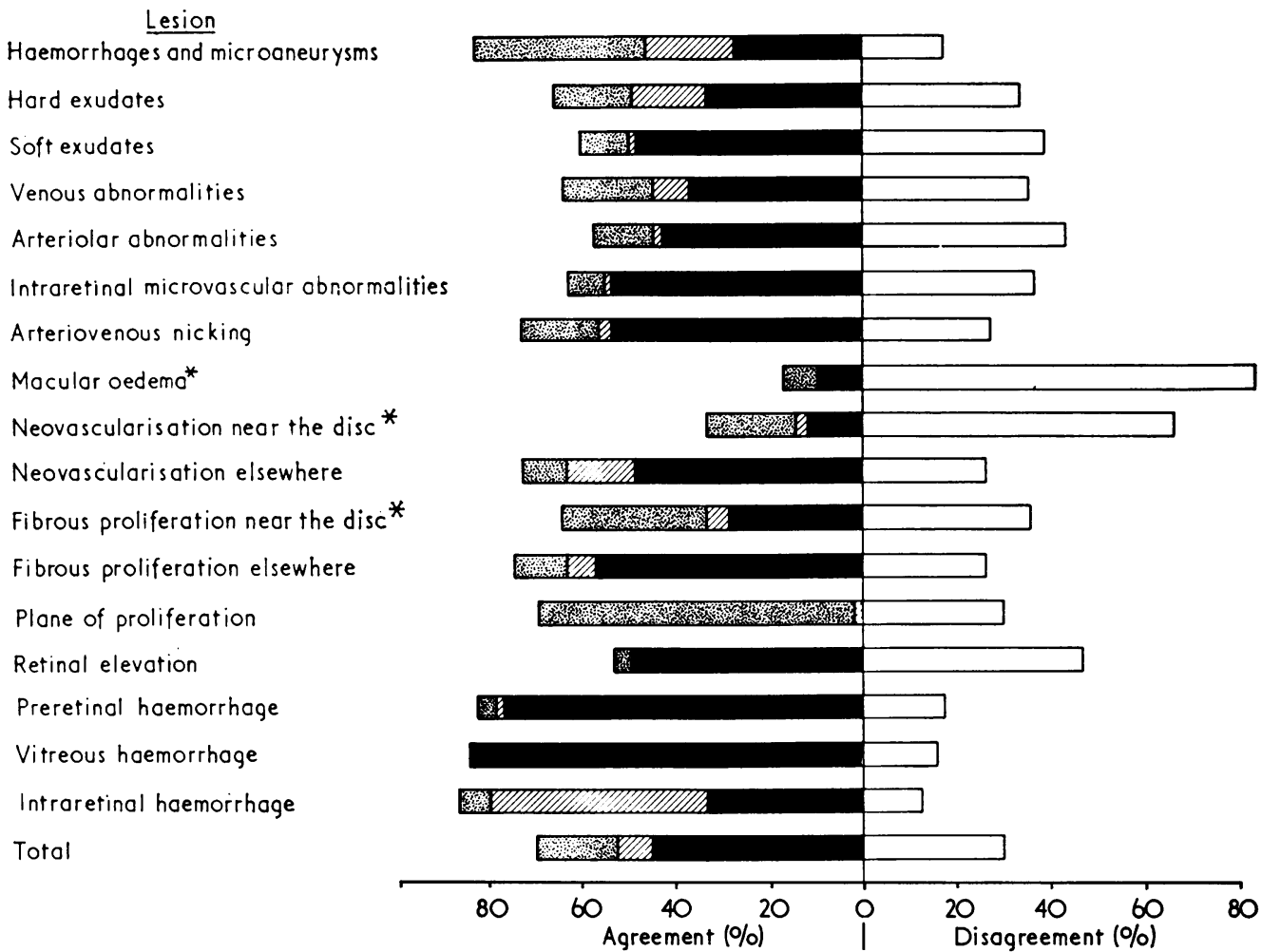

Fig. 1 Interobserver variability among 4 graders of 148 fundus photographs for 17 diabetic retinopathy lesions according to percentage of 148 photographs where the readers:

(a) all agree on a negative reading (solid bar); (b) all agree on a positive reading (striped bar); (c) give readings on two adjacent grades (dotted bar); and (d) disagree by more than one grade (clear bar)

*Percentages based on only those photographs (21) which include the appropriate field (disc or macula).

lay reader (columns 3 through 6 ) by at least $10 \%$ for 5 lesions-soft exudates, intraretinal microvascular abnormalities, neovascularisation elsewhere, retinal elevation, and preretinal haemorrhage-with maximum exceedance $51 \%$ for retinal elevation. Conversely, the best agreement between a lay reader and either physician exceeds the agreement between physicians by at least $10 \%$ for three lesionsmacular oedema, neovascularisation, and fibrous proliferation near the disc-with maximum exceedance of $48 \%$ for neovascularisation near the disc.

AGREEMENT WITHIN ONE GRADE

Fig. 1 shows for each lesion the percentage of photographs in each of 4 agreement-disagreement categories, averaged over the two replicate readings: $(a)$ all readers agree on a negative reading; (b) all readers agree on a positive reading; (c) all readings are of two adjacent grades; and $(d)$ disagreement by more than one grade. Most categories, and in particular category $d$, were quite similar for the two replicates. Agreement for all lesions combined was $70 \%$, ranging from $88 \%$ (intraretinal haemorrhage) to $17 \%$ (macular oedema). Agreement exceeded $70 \%$ in the grading of 8 of 17 lesions.

Fig. 2 shows for each lesion the percentage of cases of agreement in the first replicate reading for which each reader was a contributor to the disagreement. Results for the second replicate reading were similar except as noted below. The relative contribution to disagreement was essentially the same for the four readers in 3 lesions: arteriovenous nicking, plane of proliferation, and intraretinal haemorrhage. In no case did a physician contribute excessively to disagreement. Both lay readers contributed noticeably to disagreement in contrast to the physicians for soft exudates and retinal elevation. Reader 3 was the primary contributor for hard exudates, macular oedema, and vitreous haemorrhage disagreement; Reader 4 was so for 9 
lesions, although for 2 lesions (haemorrhages and/or microaneurysms, fibrous proliferation of disc) this result was not true for the second replicate readings. Overall, the physicians each contributed to $18 \%$ of the disagreements, Reader 3 to $27 \%$, and Reader 4 to $37 \%$.

\section{Discussion}

While absence of variability is ideal, some variation must be accepted as real in any clinical or laboratory measurement. The nature and magnitude of the variability should be assessed and presented routinely with study results, to allow users of the results to form their own judgments of the reliability of the data. General rules defining maximum acceptable variability have never been formulated; rather, the level of acceptable variability should be considered in light of the study procedures and goals. The importance of differences in performance among readers must also be evaluated in terms of the use to be made of the grades assigned to the lesions, whether in some composite score for an individual eye or on a per lesion basis, for discrimination among groups of individuals (eyes) or for assessing changes in a given eye over time.

Sources of variation in reading fundus photographs include differences in stereoviewer models (optics, illumination, interpupillary distance), reader factors (poor stereopsis, glare intolerance, fatigue threshold), and interaction between photograph quality and reader, in addition to the variability of reader judgment. In some instances the 'unknown' lesion was difficult to evaluate, or was intermediate between two standards and could easily have been given either adjacent grade. The training of the readers was designed to minimise this variation, but how well this was achieved could not be determined. Reader variability includes a contribution from these and other unrecognised factors, the resultant effect being one of overall variability in grading, usually without readily discernible components that may differentially affect readers.

Intraobserver variability as measured by weighted kappa and percent agreement showed physician readers to be less variable overall than lay readers. Soft exudates and retinal elevation were clearly marked as requiring additional training for both lay readers. Assessment by weighted kappa suggested a need for additional lay reader training in 7 lesions which had at least $10 \%$ difference between physician and lay readers, and pointed out specific areas of weakness for all readers (e.g., macular oedema and intraretinal microvascular abnormalities for the ophthalmologist). For Reader 4 other lesions were marked for improvement in reliability of grading relative to the other readers, by one or both measures of variability: haemorrhages and/or microaneurysms, intraretinal microvascular abnormalities, neovascularisation of the disc, neovascularisation elsewhere, fibrous proliferation of disc, and plane of

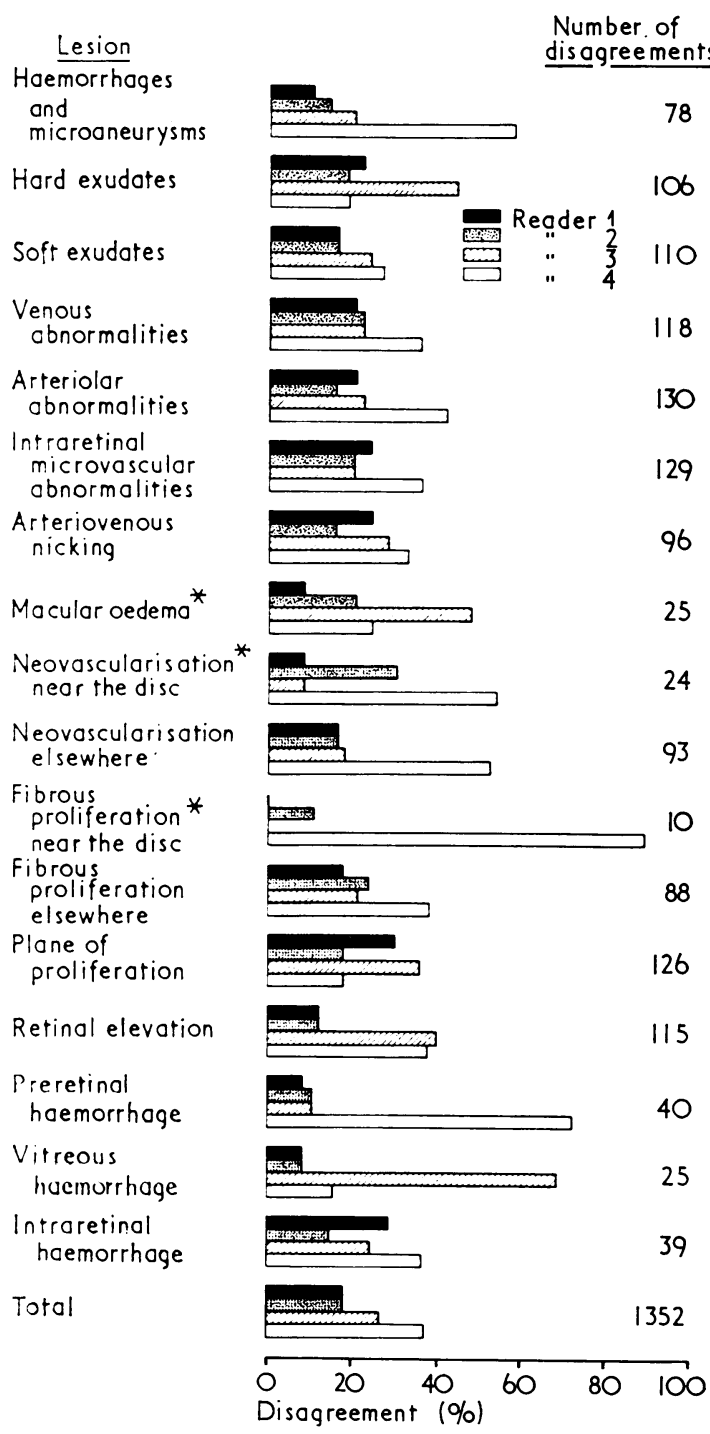

Fig. 2 Relative contribution to disagreement by each reader for 17 diabetic retinopathy lesions, for first replicate readings of 148 photographs. Readers 1 and 2 are physician readers; Readers 3 and 4 are lay readers. Disagreement includes cases where the range of readings for the four readers is greater than one grade, and cases where three readers agree on a grade but the fourth differs by one grade

*Based on only those photographs (21) which include the appropriate field (disc or macula). 
proliferation. The intraobserver variability observed in this study is probably less than would be found if the replicate slides were graded after an interval of time.

Physician-lay reader differences, although statistically significant, may often be of a magnitude to which it is difficult to assign physiological or practical importance. Nevertheless, when these differences are considered for all readers, the range of variability observed for a particular lesion may be of sufficient concern to attempt reduction through additional training or modification of the protocol; or to accept the fact that perhaps some lesions do not readily submit to a repeatable grading system and should be dropped from the study.

Percent disagreement as given in Fig. 1 is perhaps more useful because it permits assessment of the ioint performance of all four readers, for each lesion. While macular oedema and neovascularisation of disc are also clearly marked as showing excessive disagreement among readers, this measure also points to at least seven other lesions where additional attention and training would be required to achieve desirable operational agreement. Analysis as shown in Fig. 2 permits a more detailed discrimination among readers in terms of requirements for additional training and would be most useful in refinement of the grading protocol before actual application to clinical or epidemiological data. .

In addition to determining the intraobserver and interobserver variability of physicians and lay readers in grading fundus photographs for diabetic retinopathy part of the intent of this study was to determine the level of training required in order to train lay readers adequately to evaluate and grade stereo fundus photographs for particular abnormalities. For this purpose two individuals with different backgrounds and ability were selected. Reader 3 was a college graduate who was an executive secretary. Reader 4 was a statistical clerk with a high school education and trained in data processing. Although both individuals dealt peripherally with ophthalmic terminology, neither had any basic knowledge of either normal or morbid ocular anatomy. In spite of the minimum amount of training both lay readers became reasonably skilful in grading lesions. Although additional training for the readers and lesions identified with high variability was not undertaken as would normally have been done if production grading were the goal of the study, it is reasonable to assume that such training would be beneficial.

Grading of fundus photographs by lay readers has distinct advantages over the use of physicians. In addition to freeing valuable physician time, welltrained lay readers may be more systematic, objective, and conscientious. The ophthalmologist (J.P.G.) indicated that he hurried through the slides in order to get on to more interesting pursuits. This no doubt increased his variability.

Lay readers were less efficient, requiring 2 to 4 times longer to read the slides as compared to physician readers. This time should decrease with increased training and experience. Shorter physician reading time must be balanced by the fact that physician cost per reading is higher than for lay readers, and that physicians could be more effectively employed in other areas equal to their training. Adequate checks can be used to monitor lay reading, such as random samples of photographs independently read by physicians, and replicate readings.

Certain abnormalities of diabetic retinopathy were found to be more difficult to evaluate and grade than others, emphasising the need for continuing surveillance to identify weak areas early and attempt to strengthen them. One lesion in particular, macular oedema, was the source of large reading variation for both physicians and lay readers, probably due to the technique used in this study for reading the photographs. The stereo slide pair was removed from the plastic filmholder and placed in the Donaldson stereoviewer, which had been modified to handle independently mounted $35-\mathrm{mm}$ slides. The distance between the slide pair could vary in the holder. A wide separation of the slides at the extreme of fusion often gave a sensation of macular elevation which might be interpreted as macular oedema. Similarly, variation in slide separation also gave varying degrees of retinal elevation and planes of fibrous proliferation.

Lay readers had particular difficulty with fundus pathology not covered in the protocol or training slides: chorioretinal lesions, disciform macular degeneration, vein occlusion, etc. They also took the written protocol very literally. For example, arteriolar strands were described as thin white lines. An unknown slide which had a somewhat prominent straightened choroidal pattern was misclassified by one of the readers as arteriolar abnormalities. In training lay readers it is necessary to have standard photographs covering a wide range of normal anatomy and of pathological conditions other than diabetic retinopathy to help them differentiate diabetic abnormalities from other non-diabetic changes.

Although interobserver agreement is not entirely synonymous with accuracy, observer agreement is a major component. In the absence of an absolute standard to determine accuracy the subjectivity in a grading scheme such as used in this study demonstrates that accuracy may be a difficult concept to 
describe. Agreement between physicians is for some lesions less than agreement between lay reader and physician, e.g., macular oedema, and neovascularisation and fibrous proliferation near the disc. Several methods have been devised to handle observer disagreements, such as adjudication by a third reader when two readers disagree, or by calculating the mean or median of two or more independent readings. Discussion of these approaches and the definition of accuracy is beyond the scope of this study.

Mr Harold A. Kahn, Office of Biometry and Epidemiology, National Eye Institute, suggested this study and provided help and encouragement during its conduct; Alma Green and Frances Cryan participated as lay readers; Dr Howard Leibowitz, Boston University Ophthalmology Department, provided training slides; and Dr Matthew Davis, University of Wisconsin Ophthalmology Department, provided the basic grading system, standard fundus photographs of diabetic retinopathy, and study slides.

\section{Appendix: Weighted kappa}

\section{RATIONALE}

A common index of agreement for comparing the performance of 2 readers, or of 1 reader with himself, when classifying items, is the proportion of the total number of observations that are in agreement. However, in conditions such as vitreous haemorrhage, which occurs infrequently, the large number of 'absent' grades, on which there is usually little disagreement, will give an artificially high index of agreement. This index of crude agreement can be further misleading, since quite dissimilar patterns of agreement can give rise to identical measures of agreement. The Rogot-Goldberg (R-G) index of adjusted agreement, on the other hand, reflects more of the influence of individual sub-categories on the total agreement, giving more weight to smaller numbers and diminishing the effect of the larger groups. This index can be used to compare repeated readings by the same observer, or readings of 2 different observers.

The number of points in the ordinal scale used to grade a lesion varied by lesion. Further differences in the ordinal scales were created when scale values were grouped to ensure that all marginal totals (or frequencies) were non-zero, as required for computation of the R-G index. The codes for 'wrong field' and 'not determinable' were omitted as being non-ordinal, with what is judged to be a negligible effect on the analyses. The 'absent' and 'questionable' codes were combined in all lesions, since 'questionable' rarely occurred. The result of this grouping was categorisation into 2- to 4-point ordinal scales.

The chance value of the R-G index, the percentage agreement expected under total independence of the 2 observers or observations being compared, is $1 / \mathrm{L}$ for an L-point scale. Since the magnitude of departure from chance agreement is relevant in evaluating both the strength of agreement and the difference between two indices, an adjustment for departure from chance is required. This is accomplished by weighted kappa, $\kappa_{w}$, the proportion of weighted agreement corrected for chance. A further benefit from this adjustment is that the sampling distribution of $\kappa_{\mathrm{w}}$ is approximately normal for large samples (Cohen, 1968; Fleiss, Cohen and Everitt, 1972).

\section{COMPUTATION}

When $n$ subjects or items are classified by each of 2 observers on an L-point scale, agreement between the 2 classifications may be based on the data as summarised in an $\mathbf{L} \times \mathbf{L}$ table of observed proportions, with entry $\mathbf{p}_{1 j}$ the proportion of items classified by observer (1) into category $i$ and by observer (2) into category $j, \Sigma \Sigma_{i j}=1$. Crude agreement is the measure $p_{0}=\Sigma p_{i 1}$.

For a specified set of weights $\left\{w_{i j}\right\}, 0 \leqslant w_{i j} \leqslant 1, p_{0}^{*}=$ $\Sigma \Sigma w_{i j} p_{i j}$ is a weighted observed proportion of agreement. The weighted proportion of agreement expected under total independence of the two classifications is $p_{e}^{*}=\Sigma \Sigma w_{i j} p_{i} p_{. j}$, where $p_{i}$. and $p_{. j}$ are column and row marginal proportions. Weighted kappa, or weighted agreement chance corrected, is defined as $\kappa_{\mathrm{w}}=\left(\mathrm{p}_{\mathrm{o}}^{*}-\mathrm{p}_{\mathrm{e}}^{*}\right) /$ $\left(1-p_{e}^{*}\right)$ and has approximately a normal distribution with large sample variance given by Fleiss, Cohen and Everitt (1972).

The Rogot-Goldberg index of adjusted agreement (Rogot and Goldberg, 1966) is $p_{0}^{*}$ with the weights $w_{i i}=$ $\left(1 / p_{i}++1 / p_{. i}\right) / 2 L, w_{i j}=0$ for $i \neq j$. In this case, $p_{e}^{*}=1 / L$ is expected, or chance agreement.

\section{EXAMPLE}

From Table 1, for Reader 1 the $4 \times 4$ table of observed proportions $p_{i j}$ and marginal proportions based on first and second readings of the photographs according to an $\mathrm{L}=4$ point scale is as follows:

\begin{tabular}{|c|c|c|c|c|c|c|}
\hline & $\begin{array}{l}\text { First rec } \\
\text { Grades: }\end{array}$ & ding & 2 & 3 & 4 & Total \\
\hline & 1 & $53 / 148$ & $1 / 148$ & 0 & 0 & $54 / 148$ \\
\hline econd & 2 & $2 / 148$ & $18 / 148$ & $11 / 148$ & 0 & $31 / 148$ \\
\hline eadin & & 0 & $4 / 148$ & $44 / 148$ & 0 & $48 / 148$ \\
\hline & 4 & 0 & 0 & $6 / 148$ & $9 / 148$ & $15 / 148$ \\
\hline & Total & $55 / 148$ & $23 / 148$ & $61 / 148$ & $9 / 148$ & 1 \\
\hline
\end{tabular}

Crude agreement: $\quad 124 / 148=0.84$

Rogot-Goldberg (R-G): $\mathrm{p}_{0}^{*}=0.82$

Expected agreement: $\quad \mathrm{p}_{\mathrm{e}}^{*}=\mathbf{0 . 2 5}$

Weighted kappa: $\quad \kappa_{\mathrm{w}}=(0.82-0.25) / 0.75=0.76$

\section{References}

Cohen, J. (1968). Psychological Bulletin, 70, 213.

Davis, M. D., Norton, E. W. D., and Myers, F. L. (1969). In Symposium on Treatment of Diabetic Retinopathy, pp. 7-22. Edited by M. F. Goldberg and S. L. Fine. Public Health Service Publication No. 1890, US Government Printing Office: Washington, DC. 
Diabetic Retinopathy Study Research Group (1976). American Journal of Ophthalmology, 81, 1.

Fleiss, J. L., Cohen, J., and Everitt, B. S. (1972). Psychological Bulletin, 72, 323.

Ganley, J. P., and Comstock, G. W. (1973). American Journal of Ophthalmology, 76, 19.

Kahn, H. A. (1973). American Journal of Ophthalmology, 76, 164.

Kahn, H. A., et al. (1975). American Journal of Ophthalmology, 79, 768.
Koran, L. M. (1975a). New England Journal of Medicine, 293, 642 .

Koran, L. M. (1975b). New England Journal of Medicine, 293, 695.

Kupfer, C. (1973). Sight-Saving Review, 43, 17.

Landis, J. R., and Koch, G. G. (1975). Statistica Neerlandica, 29, 151.

Rogot, E., and Goldberg, I. D. (1966). Journal of Chronic Diseases, 19, 991. 